\title{
Quantifying Malware Evolution through Archaeology
}

\author{
Jeremy D. Seideman ${ }^{1 *}$, Bilal Khan ${ }^{2}$, Cesar Vargas ${ }^{3}$ \\ ${ }^{1}$ The Graduate School and University Center (CUNY), New York, USA \\ ${ }^{2}$ Department of Math and Computer Science, John Jay College (CUNY), New York, USA \\ ${ }^{3}$ NacoLabs Consulting, LLC, New York, USA \\ Email: "jseideman@gradcenter.cuny.edu, bkhan@jjay.cuny.edu, cesar@nacolabs.com
}

Received 10 January 2015; accepted 27 March 2015; published 31 March 2015

Copyright (C) 2015 by authors and Scientific Research Publishing Inc.

This work is licensed under the Creative Commons Attribution International License (CC BY).

http://creativecommons.org/licenses/by/4.0/

(c) (i) Open Access

\begin{abstract}
Dynamic analysis of malware allows us to examine malware samples, and then group those samples into families based on observed behavior. Using Boolean variables to represent the presence or absence of a range of malware behavior, we create a bitstring that represents each malware behaviorally, and then group samples into the same class if they exhibit the same behavior. Combining class definitions with malware discovery dates, we can construct a timeline of showing the emergence date of each class, in order to examine prevalence, complexity, and longevity of each class. We find that certain behavior classes are more prevalent than others, following a frequency power law. Some classes have had lower longevity, indicating that their attack profile is no longer manifested by new variants of malware, while others of greater longevity, continue to affect new computer systems. We verify for the first time commonly held intuitions on malware evolution, showing quantitatively from the archaeological record that over $80 \%$ of the time, classes of higher malware complexity emerged later than classes of lower complexity. In addition to providing historical perspective on malware evolution, the methods described in this paper may aid malware detection through classification, leading to new proactive methods to identify malicious software.
\end{abstract}

\section{Keywords}

Malware, Classification, Evolution, Dynamic Analysis

\section{Introduction}

When performing analysis on malicious software, or malware, it is important to be able to group similar mal-

${ }^{*}$ Corresponding author. 
ware samples together. In biology, organisms are classified based on genetic makeup and physical characteristics, and this classification is seen as the first task that must be done before any sort of research can be performed [1]. Sometimes malware is considered to be similar to a biological organism, although this is subject to debate [2] [3], so this concept can be extended in an attempt to perform the same operation on malware samples. Malware classification is the term used to describe the separation of malware samples into similar groups, or families. There have been attempts to standardize classification, although they have been largely unsuccessful on a large scale [4] [5].

Biological classification has an accepted and standardized taxonomy [1], with specifically defined rules for how an organism can be named and classified. While there may have been multiple systems of classification in the past, this is no longer the case; scientific bodies exist to govern the requirements for classification. Despite the lack of formal oversight in the world of malware, it is still possible to use classification techniques as part of malware analysis [6].

Malware classification is something that can be customized; each analyst's requirements can be different. If an analyst can also communicate the rationale behind the classification scheme as well as the source of those groups, then one person's classification scheme can be useful to someone else. It is for this reason that many different anti-virus software packages classify the same malware sample differently, whether it names it differently or groups it in a different family than some others. It is important to note that better classification leads to better detection [7].

This paper is organized as follows. Section 2 explores prior and related work in the field. Section 3 explores how our malware corpus was collected and analyzed. Section 4 shows how our classification scheme was used to analyze our corpus. Finally, Section 5 will examine what can be done with these results.

\section{Background}

Malware classification has been a long-studied topic with several facets that can be examined. Furthermore, classification depends on detection and analysis methods. There are generally two main types of malware analysis-static analysis, by which malware samples are examined as they exist, for example as a file on disk, and $d y$ namic analysis, whereby malware samples are examined in the course of execution. An example of static analysis would be a byte sequence signature-based detection method used in many anti-virus software packages [[8] Ch.11]. Static analysis techniques are weak against many types of polymorphism and can be weak against variants of malware presenting different signatures [9]. Dynamic analysis techniques can be more time-consuming and can be weak against techniques by which some malware samples that can detect that they are virtualized [10] but generally provide a more detailed analysis. There are several tools that could be used for dynamic analysis, such as the Norman Sandbox [11], which allows for safe execution of samples in a restricted environment. Norman Sandbox is by no means the only tool that could be used for this sort of analysis; alternatives include Alcatraz [12] and other custom tools built from virtualization software [13]. We chose Norman Sandbox due to its ability to run within Windows or GNU/Linux based systems to provide human-readable reports.

There are many ways to gather up a corpus of malware samples. The use of honeypots, such as with Honeynet [14], Nepenthes [15], its successor Dionaea [16] or other automated capture systems can be very advantageous as it makes it easier to gather a variety of samples as they live, however that method can be time-consuming, and requires network bandwidth, while also assuming that the systems utilizing these tools will be attacked and exploited. There also exist online repositories of malware samples, such as Offensive Computing [17] and VX Heavens [18], websites that host a large collection of various types of malware, spanning many types, target operating systems, and modes of operation, which provide on-demand downloading of specific samples. The advantage to this approach is that samples that have already been collected and in most cases identified are then readily available for continued analysis.

Bailey et al. [4], created a classification scheme that used malware behavior as a basis, although their scheme used a pre-processed profile that was compared using Normalized Compression Distance, looking for information overlap in the description of behavior. Our scheme abstracts descriptions of behavior into definite classes. However, since there is so much variation in classification and labeling [5], it is difficult to see how malware families are related to each other. The idea of a classification scheme is to easily group related malware together and while there are several possible alternatives that would allow this, no one has reached wide-spread prominence. 


\section{Method}

In order to define a malware class using a string of boolean values, we first must take a corpus of malware samples and then analyze them in such a way that we have information that can be represented as such. This can be broken down into the following steps:

1) Sample Acquisition and Filtering

2) Dynamic Analysis

3) Selection of Examined Characteristics

4) Boolean Profile Bitstring Construction

\subsection{Sample Acquisition and Filtering}

The basis of our malware corpus was a snapshot of the samples available from VX Heavens [18]. While they provide an "on-demand" corpus of malware that is readily accessible and downloadable, they also provide a snapshot file of a portion of their entire collection. This snapshot similarly samples the various malware types and modes, allowing a more manageable collection of samples with which to work. All samples in this collection were tagged with Kaspersky Anti-Virus and named accordingly.

We then further refined our set by eliminating those samples for which we could not determine a discovery date. This was done by examining a database of malware discovery dates created by Symantec [19], after retagging the samples with VirusTotal [20], a service that performs multiple scans on a sample with many different scanning engines. By refining our set of samples as such, we were able to perform time-based analyses of the malware, such as an examination of how long various malware classes existed.

\subsection{Dynamic Analysis}

In order to create the string of boolean values that is used to encode a sample's behavior, we first must observe how the sample affects the system upon which it runs. This is a form of dynamic analysis, in that we are observing the effects of a malware sample through its execution. In order to safely examine the samples, we employed the Norman Sandbox [11], a tool employing so-called "sandbox" technology (i.e. an isolated, protected environment in which we can execute malware samples without fear of harm to the host system) to produce a report of the sample's activity.

One of the advantages of Norman Sandbox is the degree of customization that is possible in terms of the behavioral characteristics and the output that is produced. The initialization file provides several categories of behavior, which can be included or suppressed. Our method included all behavior, with the intent of filtering out the specific items that we wanted to examine at a later time. Execution of a malware sample yielded several output files including any files that were downloaded or created as part of execution, the results of the application's scan of the sample, and a report of the behavior observed. This report was output in XML format to allow for easy text processing.

This customization also allowed us to, in advance, enumerate all of the possible behavior tags that could be output. At this point, we knew what XML tagging would be used for a variety of behaviors, and could then customize which tags we wanted to examine to create our bitstrings.

\subsection{Selection of Examined Characteristics}

While it is possible to select any number of characteristics to make up the bitstring, depending on what sort of characteristics are to be analyzed, we selected all categories of behavior that are detected by Norman Sandbox.

For our method, though, it is important to choose only characteristics that can be represented as a Boolean value-we are not concerned as much with the specifics of behavior, but rather indications of such behavior (i.e. for the purposes of this analysis, we are not as concerned with the exact files written to the hard disk as part of infection, but rather the fact that the infection causes file writing). This is because variants of a malware sample may not present the same exact behavior in terms of specifics, but may perform the same kind of task. We often see malware write random file names that change from infection to infection, as indicated by technical details in the Symantec Threat Explorer [21]; looking for the same file name would be as problematic as using only malware signatures for detection [2].

Taking the reports generated by Norman Sandbox, we then refined the results to provide, for each sample, a 
"Boolean Profile” of that sample's behavior. The profile consists of the collected boolean values for each behavioral characteristics that we analyzed.

\subsection{Boolean Profile Bitstring Construction}

In order to create our bitstring, we first must determine an order of the bits. This is an arbitrary decision; by setting one bit more "significant" than another we are not assigning it greater importance; we are merely solidifying an order of characteristics so that we can view the results in a consistent way.

In our case, we took eleven characteristics, and placed them in the following order:

1) File System-including file reads, writes, and changes

2) Registry-reading, adding, deleting, and changing registry keys

3) Network-network activity

4) Security-changes to system security

5) Process-creation of processes in memory

6) Setting Changes-altering system settings

7) Network Services-use of network services such as HTTP connections or irc connections

8) Spreads via Email-propagates using email

9) Spread LAN/WAN-propagates over the network

10) Spread Infector-propagates by infecting other files

11) Spread P2P-propagates using peer-to-peer networks

and used the characteristics in this order to create our class description bitstring.

By taking these boolean values, in this order, and concatenating them together, we were left with a binary number. This binary number, or its decimal equivalent, can be used to describe the malware sample. It is often more concise to refer to a sample by its decimal class designation rather than a binary number, especially if more bits are involved. In our scheme, we used eleven bits, so there were $2^{11}$, or 2048 possible values of the bitstring, and therefore 2048 possible classes that we could have observed.

It is important to remember that there are several variables at play in this classification scheme. First, the choice of analyzer must be taken into account due to how it reports behavior. The categories that are reported, and those that are saved, are also important. Next, the number of bits that we select and the categories that they represent are to be taken into account as well as the order in which they are displayed.

Therefore, our samples are classified by a bitstring that is valid specifically for the scanner which we use, the characteristics we search for, and the order of the bits. It is important to note that malware samples that exhibit the same of one behavior property may not end up in the same class-for example, if you have a sample that exhibits Registry behavior, and another sample that exhibits both Registry and Network behavior, these two samples will not be grouped together. Even though they share the Registry behavior, their behavioral profiles are not the same and therefore are in different groups of our classification scheme.

\section{Results}

From our collection of boolean profiles, we determined the number of samples that were in each class. Figure 1 shows the number of samples in each class. Many of the classes show almost no members; there were 116 classes that did. A list of the top 20 is shown in Table 1.

The largest class that is populated is the 0 class (00000000000), indicating samples that did not produce any behavior that was readable by the Sandbox application. This does not mean that these samples had no effect; some of them are Boot-sector viruses that do not affect the operating system directly, or samples that are targeting older operating systems that were not emulated by the Sandbox. Incidentally, there were far more samples in this class than we would have expected. Examining the samples within this class we do, in fact, see some Boot-sector viruses, and some that exploit vulnerabilities in Linux, we also found many samples that did not fit into either of those patterns. One thing we saw were many samples that infect through applications, such as through scripting, exploits in applications, Word macros, or other types of malware that requires some host other than the operating system to function. This sort of behavior would not be seen by the Sandbox as it does not emulate all of those applications. Another thing we saw were some samples that had variants in other classes. In other words, there were samples in Class 0 that were definitely related to samples within other classes. The most probably explanation of this was that whatever exploit, vulnerability, or method of infection that the sample in 


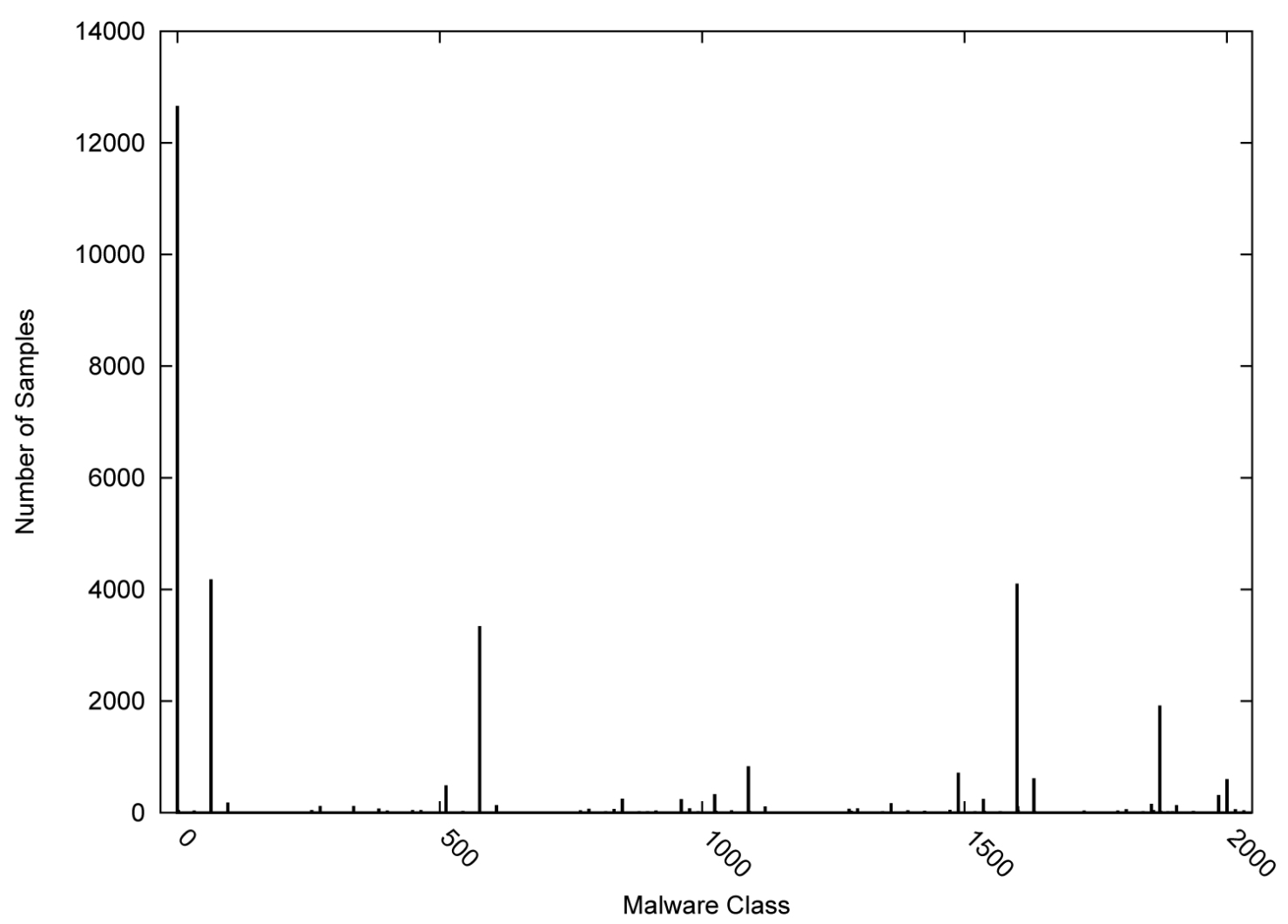

Figure 1. Frequency of class membership ( $\mathrm{x}$ = class bitstring number).

Table 1. Top 20 Malware classes in our corpus as defined by our bitstring scheme, ordered by class membership.

\begin{tabular}{cccc}
\hline Class ID & Binary Bitstring & Number of Samples & Frequency \\
\hline 0 & 00000000000 & 12,639 & 0.388020753384705 \\
64 & 00001000000 & 4156 & 0.127590335553986 \\
1600 & 11001000000 & 4080 & 0.125257114788322 \\
576 & 01001000000 & 3321 & 0.101955607404906 \\
1872 & 11101010000 & 1897 & 0.0582384183219231 \\
1088 & 10001000000 & 810 & 0.0248672213182697 \\
1488 & 10111010000 & 694 & 0.0213059896233076 \\
1632 & 11001100000 & 593 & 0.018205262026832 \\
2000 & 11111010000 & 578 & 0.0177447579283456 \\
512 & 01000000000 & 467 & 0.0143370275995456 \\
1024 & 10000000000 & 307 & 0.00942498388235655 \\
1984 & 11111000000 & 295 & 0.00905658060356737 \\
848 & 01101010000 & 229 & 0.00703036257022688 \\
1536 & 11000000000 & 222 & 0.00681546065759985 \\
960 & 01111000000 & 219 & 0.00672335983790256 \\
96 & 00001100000 & 162 & 0.00497344426365395 \\
1360 & 10101010000 & 147 & 0.00451294016516747 \\
1856 & 11101000000 & 139 & 0.00426733797930802 \\
608 & 01001100000 & 113 & 0.00346913087526479 \\
1904 & 11101110000 & 113 & 0.00346913087526479 \\
\hline
\end{tabular}


Class 0 used was closed; the Sandbox therefore represented a more modern iteration of the operating system in which some of the security problems were fixed.

By ranking the frequencies of each class, we see that the frequencies drop dramatically after the first class, after which the decrease in frequency is much less dramatic. Figure 2 shows the frequency distribution on a log scale. We see that certain classes of malware are rarer in the wild than others, although the reason for this is difficult to know. Possibilities include program complexity and the ease in which a sample within a particular class can infect computers, both of which are the subject of future research.

Examining the frequency rankings, we found that the frequencies tended to follow a power-law distribution. Our data shows that our corpus approximately follows an expected power law curve.

Class 64 (00001000000) Class 64 malware samples exhibit only Process creation. Nevertheless, we see many types of malware within this class, such as worms, download Trojans, spyware Trojans, and even some that target Linux instead of Windows. In these cases, it would appear that the process created in memory is what performed many of the tasks, often beyond the reach of the analysis tool.

Class 1600 (11001000000) Class 1600 malware exhibits File System, Registry and Process creation properties. These samples may read and write files on the computer, enumerate and alter registry settings, and create processes in memory. The changes to the file system or registry may be performed by the main program, or by a spawned process. Note that Registry operations do not necessarily imply file system changes; Norman Sandbox differentiates between the two kinds of activity.

Class 576 (01001000000) Class 576 malware exhibits Registry and Process creation properties. There are many ways that this could be leveraged as the registry is capable of storing information and code, which could be run at a later time. One way that this could happen is if the malware sample creates a process that runs, using information from the registry to act. Alternatively, the process could also be operating directly on the registry, instead of using it as a "seed" for code.

Class 1872 (11101010000) Class 1872 malware exhibits File System, Registry, Network, Process creation and Network service properties. Network and Network Services tend to go hand-in-hand-network refers to the use of network traffic by a malware sample, whereas network services refers to higher level network operations,

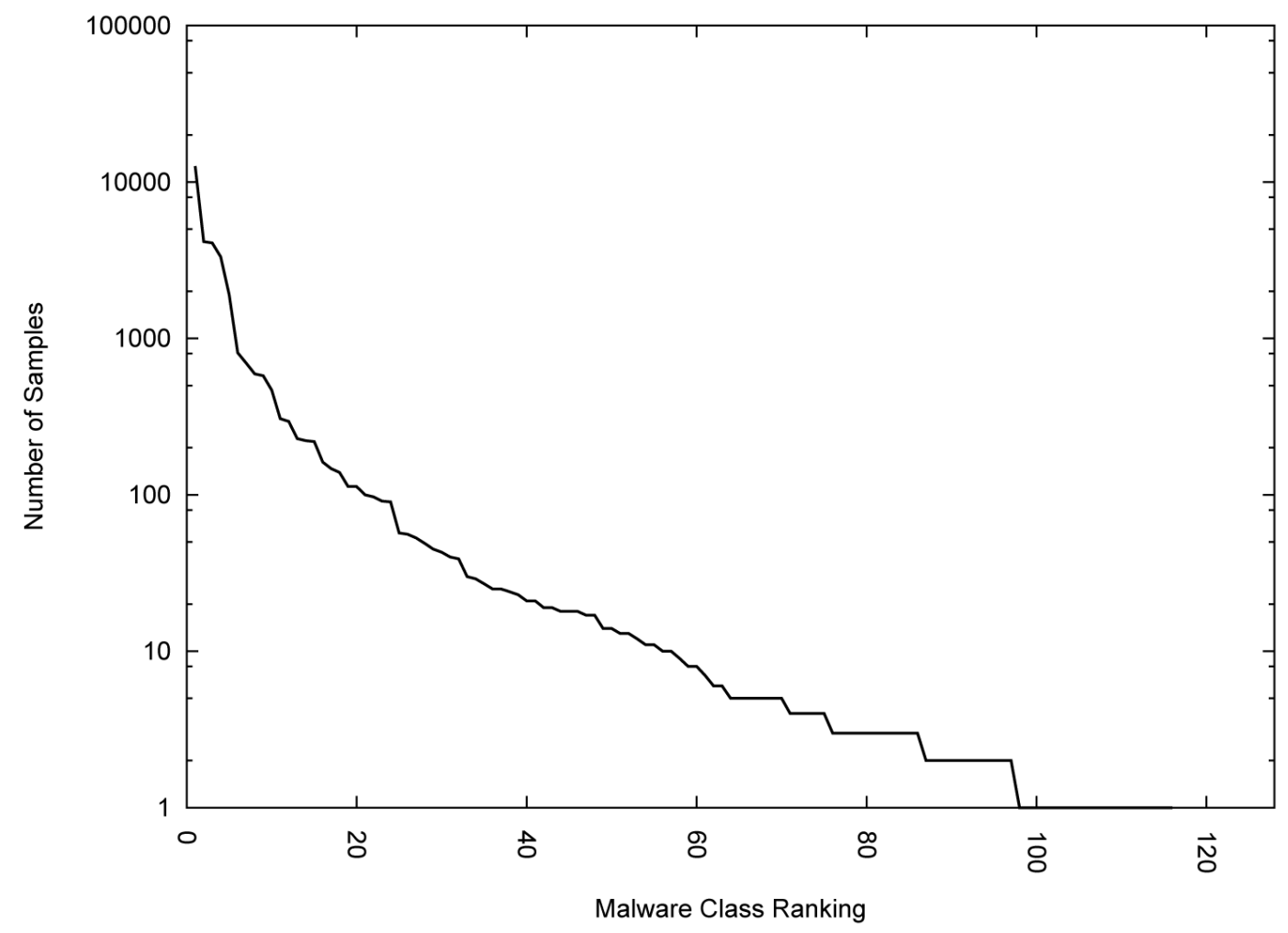

Figure 2. Frequency of class membership, on a log scale ( $\mathrm{x}=$ class bitstring rank). 
such as connecting to an IRC server or starting a web server. A sample in this class, for example, might alter the registry to allow for a program to start on boot (to ensure that its programs are always running), then connect over a network to download an executable program, which is then written to disk and run. The spawned process will then perform whatever acts it wishes, and due to the registry change, will start up on boot to continue to act upon the system.

Class 1088 (10001000000) Class 1088 malware exhibits File system and Process creation properties. Members of this class could write small programs to act on the system to disk, and then subsequently execute those programs.

\subsection{Complexity over Time}

We hypothesize that malware complexity will increase over time, both because of and in order to take advantage of the increasing complex nature of computer systems. Our hypothesis is that a more complicated piece of malware (defined as a sample that exhibits more properties than a less complex sample) will appear after simpler malware, chronologically. We do not deny the fact that a "simple" type of malware may continue to exist and infect; it is possible that some of those samples are uniquely fit to survive the changing computer landscape.

We examined malware samples in pairs from our dated corpus, using the algorithm defined in Algorithm 4.1. First, we defined two functions- ones $(X)$ which returns the string of positive boolean values in sample $X$ 's profile, including position within the string, and date $(X)$, which returns the discovery date of sample $X$ through a lookup.

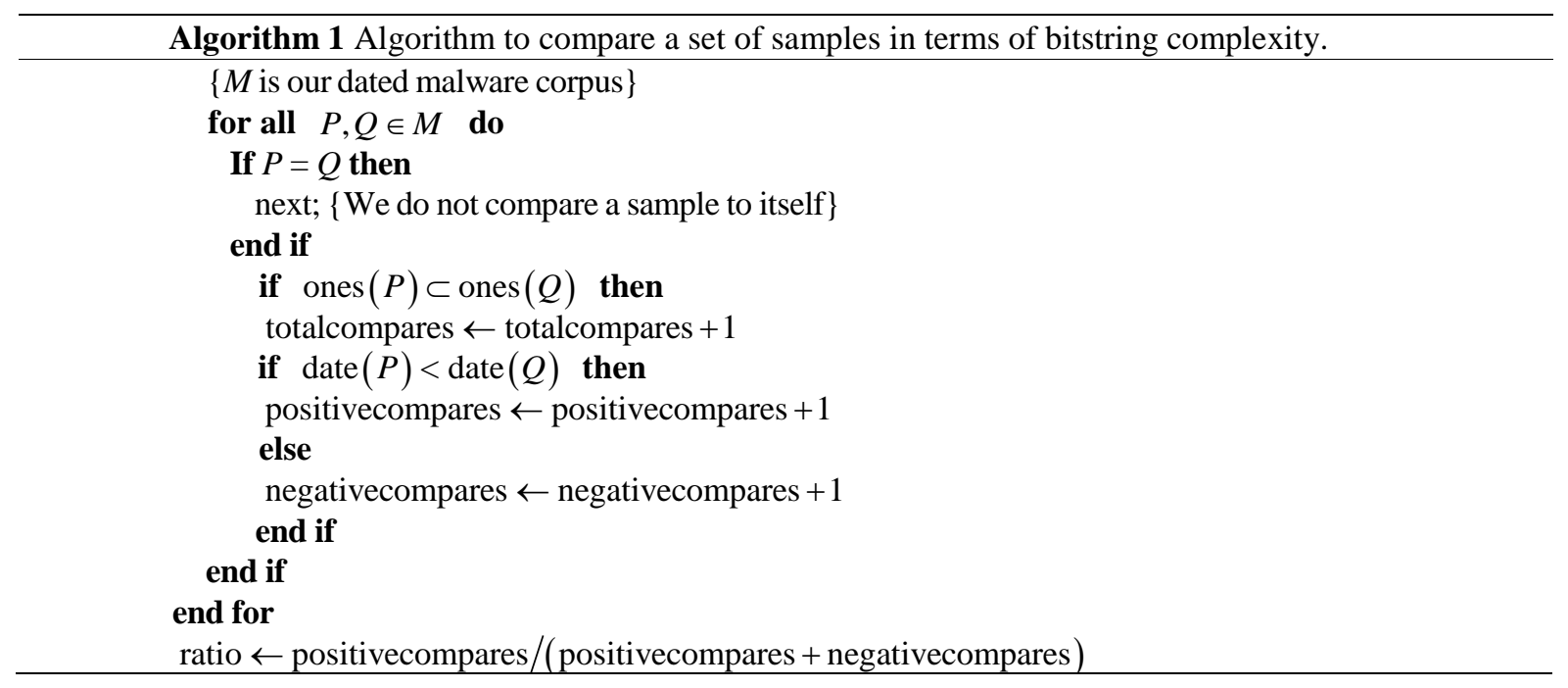

By comparing all pairs in $M$, there were over 1,000,000,000 possible pairwise comparisons. Of these comparisons, 379,950,237 pairs were checked where one sample's properties were a subset of the other. 52.4\%, or 199,113,920 comparisons supported our theory, while 180,836,317 rejected it. This result was skewed due to the differing numbers of samples within each class. This algorithm looks at any arbitrary pair of samples, so there were pairs that would be compared twice, one time which would support our hypothesis and one which would not. We then refined our measure by first filtering out samples so that we were able to determine the "progenitor" of each malware class-the sample at which a particular bitstring first appeared. This was accomplished by examining the corpus, and, for each sample, checking if its discovery date was the earliest for its respective class. If it was, we kept it as a "progenitor" of that class. At the end, we had a total of 116 samples left to check. We then ran the corpus through the same algorithm and found that, of the 13,340 possible pairwise comparisons, there were 1580 comparisons where one sample was a subset of the other. Of those, 82.7\%, or 1306 supported our hypothesis and 274 rejected it.

\subsection{Longevity of Malware Classes}

Since we have a dated corpus of malware, we can also plot a timeline of the first appearance of a sample of the 
class, and find out how long that class persists in our corpus. By examining the samples in the corpus and determining, for each class, the date of the first appearance and the date of the last one, we were able to determine the longevity of each class within our corpus. The average longevity for a malware class was approximately 1648 days, ranging from 0 days to 6438 days. A longevity of 0 days indicates that the first and last appearance of a class was on the same day, which could indicate that a particular class was ineffective at spreading, or that that particular class did not spawn many variants within the same class. It does not exclude the possibility that the class served as the basis for a more complex class, however. A higher longevity number does indicate that the class is uniquely suited for survival-while the specific exploits that different samples within the class employ may differ, the overall behavior remains constant and therefore that kind of attack that a sample in that class represents remains possible for a longer amount of time.

Table 2 and Table 3 show the longevity of the various classes, ordered by the length of time that a class was active, and the number of samples within that class, respectively. We see that for the most part, there is a correlation between the number of samples and the length of time that a malware class is active-the more samples of a class that exist, the longer that class is able to survive. This can be attributed to the fact that even if a sample is particularly virulent and has a profound effect on a computer, if new samples that are similar to it are not created, eventually anti-virus and anti-malware programs will be able to eradicate that sample and other members of its class. Without new samples being created within a class, said class will eventually die out in favor of newer, possibly more complex ones, that take advantage of more of the features available to a malware writer.

\section{Conclusions and Further Work}

Our method creates malware classification that creates a simple, human-readable classification system. The ad-

Table 2. Malware Class Longevity, ordered by class survival duration (in days).

\begin{tabular}{|c|c|c|c|c|c|}
\hline Class ID & Bitstring & Number & First Appearance & Last Appearance & Total Duration (Days) \\
\hline 0 & 00000000000 & 12,639 & 19900601 & 20080116 & 6438 \\
\hline 1600 & 11001000000 & 4080 & 19961126 & 20080702 & 4236 \\
\hline 848 & 01101010000 & 229 & 19971208 & 20080702 & 3859 \\
\hline 1088 & 10001000000 & 810 & 19971208 & 20080626 & 3853 \\
\hline 608 & 01001100000 & 113 & 19961126 & 20070220 & 3738 \\
\hline 1872 & 11101010000 & 1897 & 19971208 & 20080125 & 3700 \\
\hline 576 & 01001000000 & 3321 & 19971208 & 20080125 & 3700 \\
\hline 1280 & 10100000000 & 49 & 19971208 & 20070820 & 3542 \\
\hline 64 & 00001000000 & 4156 & 19971208 & 20070705 & 3496 \\
\hline 1120 & 10001100000 & 90 & 19971208 & 20070530 & 3460 \\
\hline 96 & 00001100000 & 162 & 19971208 & 20070530 & 3460 \\
\hline 1632 & 11001100000 & 593 & 19971208 & 20070530 & 3460 \\
\hline 512 & 01000000000 & 467 & 19971208 & 20070530 & 3460 \\
\hline 1792 & 11100000000 & 18 & 19971208 & 20070530 & 3460 \\
\hline 1488 & 10111010000 & 694 & 19971208 & 20070530 & 3460 \\
\hline 272 & 00100010000 & 100 & 19971208 & 20070322 & 3391 \\
\hline 1024 & 10000000000 & 307 & 19971208 & 20070227 & 3368 \\
\hline 1536 & 11000000000 & 222 & 19971208 & 20070220 & 3361 \\
\hline 1360 & 10101010000 & 147 & 19971208 & 20061112 & 3261 \\
\hline 1984 & 11111000000 & 295 & 19971208 & 20061107 & 3256 \\
\hline
\end{tabular}


Table 3. Malware Class Longevity, ordered by class membership.

\begin{tabular}{|c|c|c|c|c|c|}
\hline Class ID & Bitstring & Number & First Appearance & Last Appearance & Total Duration (Days) \\
\hline 0 & 00000000000 & 12,639 & 19900601 & 20080116 & 6438 \\
\hline 64 & 00001000000 & 4156 & 19971208 & 20070705 & 3496 \\
\hline 1600 & 11001000000 & 4080 & 19961126 & 20080702 & 4236 \\
\hline 576 & 01001000000 & 3321 & 19971208 & 20080125 & 3700 \\
\hline 1872 & 11101010000 & 1897 & 19971208 & 20080125 & 3700 \\
\hline 1088 & 10001000000 & 810 & 19971208 & 20080626 & 3853 \\
\hline 1488 & 10111010000 & 694 & 19971208 & 20070530 & 3460 \\
\hline 1632 & 11001100000 & 593 & 19971208 & 20070530 & 3460 \\
\hline 2000 & 11111010000 & 578 & 19971208 & 20060915 & 3203 \\
\hline 512 & 01000000000 & 467 & 19971208 & 20070530 & 3460 \\
\hline 1024 & 10000000000 & 307 & 19971208 & 20070227 & 3368 \\
\hline 1984 & 11111000000 & 295 & 19971208 & 20061107 & 3256 \\
\hline 848 & 01101010000 & 229 & 19971208 & 20080702 & 3859 \\
\hline 1536 & 11000000000 & 222 & 19971208 & 20070220 & 3361 \\
\hline 960 & 01111000000 & 219 & 19980616 & 20060814 & 2981 \\
\hline 96 & 00001100000 & 162 & 19971208 & 20070530 & 3460 \\
\hline 1360 & 10101010000 & 147 & 19971208 & 20061112 & 3261 \\
\hline 1856 & 11101000000 & 139 & 19971208 & 20060818 & 3175 \\
\hline 1904 & 11101110000 & 113 & 19971208 & 20060817 & 3174 \\
\hline 608 & 01001100000 & 113 & 19961126 & 20070220 & 3738 \\
\hline
\end{tabular}

vantage of this system is that, due to the limited number of possible classes, it is possible to create a look-up table of malware behavior; a new sample that is examined and exhibits behavior can immediately be grouped with samples that have similar behavior. At that point, further, more directed analysis can be done. The disadvantage of this method is the overhead involved with the classification; while determining boolean class is quick, since we are performing dynamic analysis, we are bound by the time it takes to perform an execution of the sample. Of course, this means that this method is also subject to some of the pitfalls of dynamic analysis; some samples are able to detect that they are being used within a sandboxed environment and can adjust their execution accordingly [2].

Going forwards, this method is most useful as a sort of pre-processing phase of a collection of malware; when comparing samples to each other we have found that often it is wasteful to compare samples that are unrelated to each other; that the relationship between malware samples when grouping them into families is facilitated when samples that are more closely related are set to be grouped together.

An extension of this method can also be used as part of a malware detection system, such as Virus Total [20] or Cloud AV [22], both of which leverage multiple approaches to increase detection percentages. Even Norman Sandbox, for example, is part of a suite of software that includes an anti-virus program; increasingly we see that using only signature-based detection leads to missed infections [2]. Being able to examine file operations before opening or execution can be helpful in determining whether or not a sample is infected; if a more complex bitstring is employed, and a file on the system exhibits the same properties as one of the classes that contains a large amount of malware, the scanning engine can then interrupt execution or at least flag the sample as suspicious, based solely on this heuristic rule. While increasing the number of Boolean values does increase the number of possible classes that may be populated, it is easy to see that the majority of them will remain emptysome classes even act as "meta-classes" of others (e.g. if a sample does not exhibit the Registry property, then any of the categories which specialize that property will also not be exhibited). 
Finally, this method can be used to classify very specific behavior, given that classification of behaviors can be as fine-grained as desired. A detector can be programmed to look for any operation within a category of behavior; if we are looking at registry changes, we can design our scheme to look for the most specific registry change we want to use as a basis of classification. This level of customization allows for more targeted detectors which, while not always useful in the real-world, are useful in an isolated setting as part of a reverse-engineering approach.

\section{References}

[1] Classification of Species, 2009. https://web.archive.org/web/20120121022919/http://classes.entom.wsu.edu/348/classification.htm

[2] Seideman, J. (2009) Recent Advances in Malware Detection and Classification: A Survey. Technical Report, The Graduate School and University Center of the City University of New York.

[3] Spafford, E.H. (1994) Computer Viruses as Artificial Life. Artificial Life, 1, 249-265. http://dx.doi.org/10.1162/artl.1994.1.3.249

[4] Bailey, M., Oberheide, J., Andersen, J., Morley Mao, Z.Q., Jahanian, F. and Nazario, J. (2007) Automated Classification and Analysis of Internet Malware. Proceedings of RAID 2007, 178-197. http://dx.doi.org/10.1007/978-3-540-74320-0_10.

[5] Riau, C. (2002) A Virus by Any Other Name: Virus Naming Practices. http://www.symantec.com/connect/articles/virus-any-other-name-virus-naming-practices

[6] Gandotra, E., Bansal, D. and Sofat, S. (2014) Malware Analysis and Classification: A Survey. Journal of Information Security, 5, 56-64. http://dx.doi.org/10.4236/jis.2014.52006

[7] Lee, T. and Mody, J.J. (2006) Behavioral Classification. Proceedings of EICAR 2006, May 2006, 1-17.

[8] Szor, P. (2005) The Art of Computer Virus Research and Defense. Addison-Wesley, New York.

[9] Jacob, G., Debar, H. and Filiol, E. (2008) Behavioral Detection of Malware: From a Survey towards an Established Taxonomy. Journal in Computer Virology, 4, 251-266. http://dx.doi.org/10.1007/s11416-008-0086-0

[10] Andreas Moser, Christopher Kr"ugel, and Engin Kirda. (2007) Exploring Multiple Execution Paths for Malware Analysis. Proceedings of the 2007 IEEE Symposium on Security and Privacy, 2007, 231-245. http://dx.doi.org/10.1109/SP.2007.17

[11] (2009) Norman Sandbox. https://web.archive.org/web/20101005091013/http://www.norman.com/technology/norman_sandbox/

[12] Liang, Z.K., Sun, W.Q., Venkatakrishnan, V.N. and Sekar, R. (2009) Alcatraz: An Isolated Environment for Experimenting with Untrusted Software. ACM Transactions on Information and System Security, 12, 1-37.

[13] Buyrukbilen, S. and Deryol, R. (2008) An Automated System for Behavioral Malware Analyis. Technical Report, John Jay College of Criminal Justice, City University of New York.

[14] (2010) The Honeynet Project. http://www.honeynet.org

[15] (2010) Nepenthes-Finest Collection. http://nepenthes.carnivore.it/

[16] (2012) Dionaea-Catches Bugs. http://dionaea.carnivore.it/

[17] (2010) Offensive Computing: Community Malicious Code Research and Analysis. http://www.offensivecomputing.net

[18] VX Heavens, 2010. http://vxheaven.org/

[19] Symantec, 2012. http://www.symantec.com/index.jsp

[20] VirusTotal, 2008. http://www.virustotal.com

[21] (2012) Threat Explorer-Spyware and Adware, Dialers, Hack Tools, Hoaxes and Other Risks. http://www.symantec.com/security_response/threatexplorer/

[22] Oberheide, J., Cooke, E. and Jahanian, F. (2008) Cloudav: N-version Antivirus in the Network Cloud. Proceedings of the 17th USENIX Security Symposium, 91-106. http://www.usenix.org/events/ 